\title{
Nuevas estrategias en el proceso de enseñanza-aprendizaje de la asignatura Experimentación Química II del Grado en Ingeniería Química Industrial
}

\author{
Elena Matilde SánCHez FernándeZ \\ Departamento de Química Orgánica \\ Facultad de Química \\ Universidad de Sevilla \\ esanchez4@us.es \\ ORCID: https://orcid.org/0000-0002-8617-8234 \\ D.O.I.: http://dx.doi.org/10.12795/JDU.2018.i01.43 \\ Pp.: $766-783$
}

\section{Resumen}

El trabajo realizado durante el desarrollo del ciclo de mejora experimentado en la asignatura Experimentación en Química II se ha centrado en dos pilares fundamentales:

1) Interconexión de los contenidos teórico-prácticos de modo que el conocimiento que se construya sea un conocimiento con cimientos sólidos, constituido por experiencias que se retroalimentan.

2) Implicación del alumno en el proceso de enseñanza-aprendizaje. El ciclo de mejora está diseñado con la idea de mantener la motivación y la participación del alumnado a lo 
largo de este proceso mediante la realización de un trabajo-esfuerzo constante que permita la asimilación de los principios básicos de la asignatura.

Palabras clave: Experimentación en Química II, Grado en Ingeniería Química Industrial, docencia universitaria, Experimentación docente universitaria, retroalimentación.

\section{Descripción del contexto}

La asignatura en la que se ha desarrollado el ciclo de mejora durante mi participación en la Red para la Formación e Innovación Docente (REFID) es Experimentación en Química II, la cual corresponde a una asignatura de carácter obligatorio (6 créditos ECTS) del segundo cuatrimestre de segundo curso del Grado en Ingeniería Química Industrial que se imparte en la Escuela Politécnica Superior (EPS) de la Universidad de Sevilla. Normalmente se encuentran matriculados en la asignatura entre 35-40 alumnos. Se dividen en 6 grupos de 6-7 alumnos cada uno. Las clases se imparten en un laboratorio que tiene capacidad para 14 alumnos. Consisten en 3 sesiones semanales de 3 horas cada sesión (9 horas a la semana). A lo largo de la asignatura se realizan una serie de prácticas de forma individual, cada alumno es responsable de su resultado. El material del que disponen es un guión de prácticas con todas las instrucciones necesarias para el desarrollo experimental. Como sistema de evaluación se cuenta con un examen teórico de los contenidos de la asignatura y de un examen práctico donde demuestren las destrezas adquiridas en el laboratorio.

\section{Diseño del Ciclo de Mejora Docente}

Las prácticas en las que he planteado los ciclos de mejora son las siguientes:

(1) Separación por extracción (6 h)

Jornadas de Formación e Innovación Docente del Profesorado | № 1 (2018) 
(2) Sintesis de $p$-nitroanilina $(9 \mathrm{~h})$

(3) Sintesis de difenilmetanol (3 h)

Comentar, en primer lugar, que he elegido estas tres prácticas para realizar los ciclos de mejora ya que he observado en años anteriores en los que he impartido la asignatura que son las que resultan más complejas para el alumnado. En general son prácticas densas, con mucha información teórica (no solo experimental), en las que se manejan muchos conceptos teórico-prácticos que no terminan de entender/dominar tal como demuestran en los exámenes. Cada una de estas prácticas resulta de la elaboración de un problema que aborda un núcleo temático (Clayden, Greeves, Warren y Wothers, 2001).

Lo primero que planteo en este ciclo de mejora es un cuestionario previo justo al comienzo de cada práctica con objeto de analizar el conocimiento inicial del alumnado (el alumno ha de haber cursado en el primer cuatrimestre la asignatura Química Orgánica también de carácter obligatorio ( 6 créditos ECTS, donde aparecen todos los aspectos teóricos que se ponen de manifiesto en esta asignatura), por tanto sus ideas iniciales no deberían partir de cero. Aunque no he eliminado el cuestionario previo de años anteriores tipo test, este año he introducido un cuestionario con 4-5 cuestiones abiertas donde ellos puedan exponer brevemente su conocimiento de partida, para así poder comprobar si hay diferencias en cuanto a los resultados cuando se enfrenten a uno tipo test o a otro tipo de cuestionario. Desde mi punto de vista, las cuestiones tipo test presentan muchas carencias y sirven para muy poco a la hora de evaluar al alumnado ya que muchas veces las respuestas son contestadas al azar. Decía Cuesta Dutari, profesor de matemáticas de la Universidad de Salamanca: "Un test es lo mismo que mirar por el ojo de una cerradura, no se ve nada y es una falta de educación" (Martín Sánchez, 2018). En el apartado de evaluación del ciclo de mejora docente se comentarán las diferencias observadas entre ambos cuestionarios. 
El mismo cuestionario que se pasa inicialmente se pasará al finalizar por completo la práctica, con lo cual podremos evaluar la evolución de los estudiantes. A continuación, se presentan los cuestionarios inicial-final para las tres prácticas elegidas.

El cuestionario inicial-final no 1 para la práctica Separación por extracción contiene las siguientes cuestiones:

P1.1. ¿Cuáles son los componentes presentes en la mezcla que se pretende separar?

P1.2. En una separación por extracción, ¿en función de qué se separan los compuestos?

P1.3. ¿El ácido salicílico es más o menos ácido que el 1-naftol? ¿Por qué?

P1.4. ¿El 1,3-dinitrobenceno presenta carácter básico? Razone la respuesta.

P1.5. ¿Para qué se utiliza la técnica de cromatografía en capa fina en esta práctica en cuestión?

El cuestionario inicial-final no 2 para la práctica Síntesis de p-nitroanilina contiene las siguientes cuestiones:

P2.1. ¿Por qué se alarga la secuencia sintética a 3 pasos cuando aparentemente se podría hacer en una única etapa?

P2.2. Formule los compuestos implicados en la práctica

P2.3. ¿De qué está compuesta la mezcla sulfonítrica?

P2.4. ¿Por qué se forma mayoritariamente p-nitroanilina frente a o-nitroanilina?

P2.5. ¿Cuándo se lleva a cabo la eliminación del grupo protector?

El cuestionario inicial-final no 3 para la práctica Síntesis de difenilmetanol contiene las siguientes cuestiones:

P3.1. ¿Qué reactivos intervienen en la preparación de difenilmetanol? 
P3.2. ¿Cómo controlaría que toda la benzofenona se ha transformado en difenilmetanol?

P3.3. Sabiendo que el difenilmetanol es un compuesto sólido, ¿cómo llevaría a cabo su purificación?

P3.4. Si el valor de Rf de la benzofenona usando como eluyente DCM:MeOH (1:1) es 0.5 y del difenilmetanol es 0.25, ¿cuál de los dos compuestos es más polar? ¿Por qué?

Una carpeta denominada boletín de ejercicios se ha incorporado este año en la plataforma virtual a disposición del alumnado. Esta carpeta contiene diferentes tipos de problemas: formulación (primordiales para abordar la asignatura), cálculos y ejercicios de diferentes situaciones de la técnica de cromatografía en capa fina, técnica usada a diario en los laboratorios de Química Orgánica (Palleros, 2000). La idea de este boletín de problemas es que ellos tengan a su disposición material con casos prácticos que puedan resolver tranquilamente, fuera del horario de clase con la finalidad de profundizar y afianzar lo que se ha visto en las diferentes sesiones de trabajo, fomentando así el trabajo, el esfuerzo y el compromiso con la asignatura. La experiencia me dice que aunque son cosas que se dan por sabidas, el alumno presenta muchas deficiencias que se reflejan en los exámenes año tras año.

\section{Mapa de contenidos}

El mapa de contenidos que se presenta para las tres prácticas es el siguiente:

Jornadas de Formación e Innovación Docente del Profesorado | № 1 (2018) Esta obra se distribuye con la licencia Creative Commons 


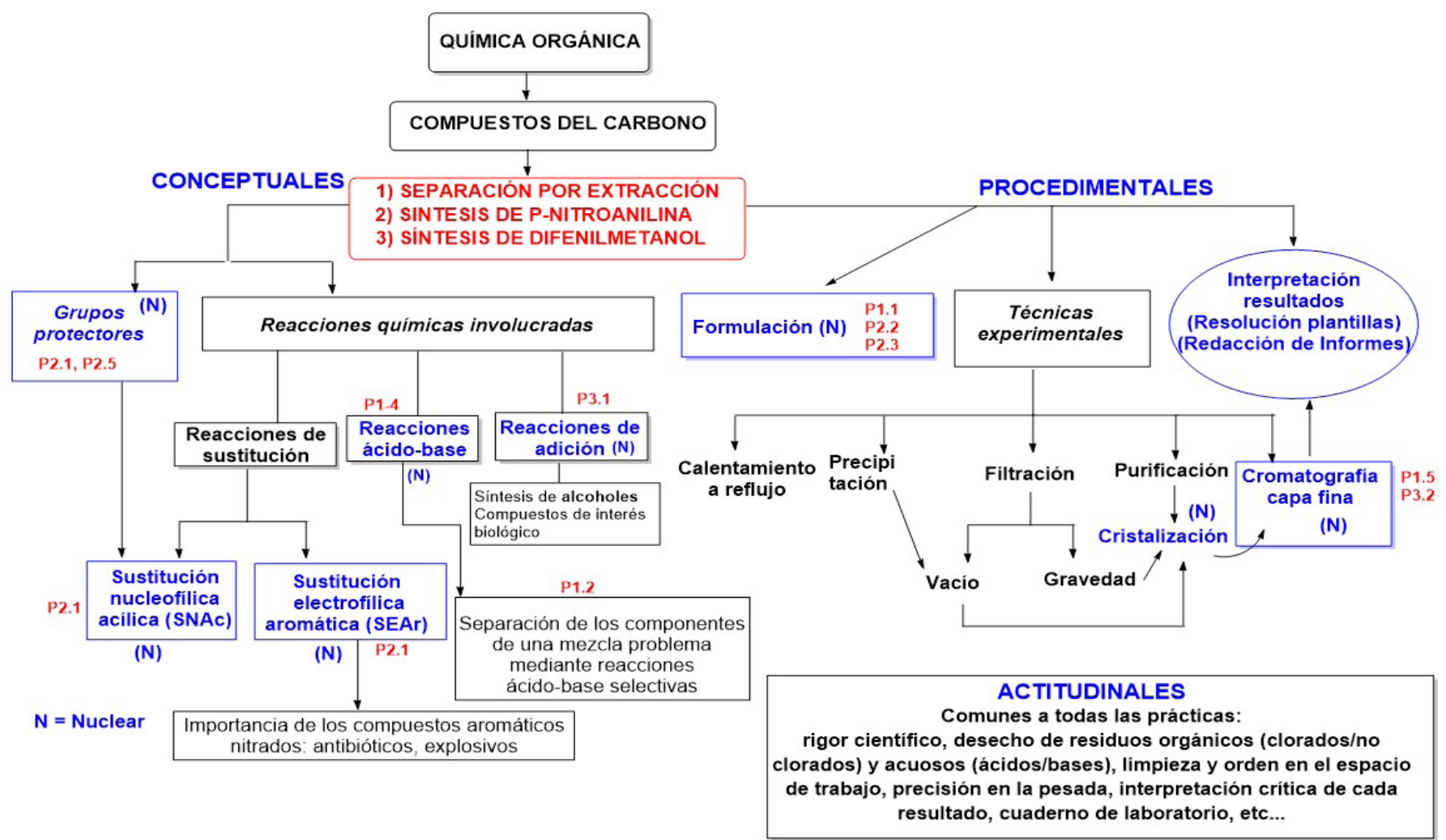

Figura 1. Mapa de contenidos correspondiente a las tres prácticas en las que se han llevado a cabo los ciclos de mejora: Separación por extracción, Síntesis de la p-nitroanilina y Síntesis de difenilmetanol.

Jornadas de Formación e Innovación Docente del Profesorado | № 1 (2018)

cc (i) $\Theta$ Esta obra se distribuye con la licencia Creative Commons 


\section{Modelo metodológico y secuencia de actividades programada}

Antes de comenzar con el modelo metodológico, comentar que este año se han incorporado como parte de un proyecto de innovación docente en el que participo titulado: Mejoras metodológicas en asignaturas del área de Química Orgánica del Grado en Ingeniería Química Industrial, nuevas plantillas para la elaboración de informes por parte del alumno. Estas plantillas recogen varias preguntas que han de ser contestadas durante la realización de las prácticas, en el laboratorio, las cuales darán idea al profesor si el alumno ha entendido el objetivo de la práctica y si ha alcanzado los conocimientos deseados. Estas plantillas sustituyen al tradicional informe que se le pedía al alumno una vez finalizada la práctica. Con estas plantillas lo que se pretende es que el alumno lleve la asignatura al día, ya que no puede empezar una práctica nueva si no se ha entregado la plantilla de la práctica anterior, incluye preguntas clave, donde han de reflejar los conocimientos fundamentales que han de haber adquirido al finalizar las sesiones de trabajo. Estas plantillas abarcan todos los contenidos que se plantean en los cuestionarios inicial-final. La idea es que aumente la implicación (constancia, dedicación, esfuerzo) del alumno en la asignatura desde el principio, no al final cuando llega la fecha límite de entrega de informes o cuando se acerca la fecha del examen. Aún así, y para no alejarnos completamente del método tradicional, se les pide la redacción de dos informes por escrito de tres-cuatro páginas.

A continuación se detalla el modelo metodológico y secuencia de actividades:

Separación por extracción (2 sesiones de 3 horas cada una)

1a Sesión (180 min)

Jornadas de Formación e Innovación Docente del Profesorado | № 1 (2018) Esta obra se distribuye con la licencia Creative Commons 
- Se colocan los alumnos en sus puestos de trabajo (gafas, guantes, bata de laboratorio...): 5 minutos.

- Cuestionario inicial (5 preguntas): 15 - 20 minutos.

- Contextualizar la práctica, planteamiento de la misma en la pizarra haciendo hincapié en la formulación y en las reacciones ácido-base implicadas que nos permitirán separar los componentes de una mezcla, en el material utilizado y en las técnicas experimentales que van a emplear a lo largo del desarrollo de la misma: 30 minutos.

Se preguntará al alumnado y se valorará su participación. Reflexión en grupo.

- Trabajo individual experimental en sus puestos de trabajo: 90 minutos. En esta primera sesión llegarán a la separación de los tres componentes y al aislamiento de dos de ellos, el tercero quedará en disolución para ser aislado en la siguiente sesión. También deberán ir completando la plantilla.

- Recapitulación en la pizarra mediante ejercicios en los que se trabaje con diagramas que representen el proceso de extracción líquido-líquido: 20 minutos.

- Desecho de residuos, limpieza del material y del puesto de trabajo: 15 min.

\section{2a Sesión (180 min)}

- Se colocan los alumnos en sus puestos de trabajo: 5 minutos.

- Aislamiento del tercer componente de la mezcla: 20 minutos.

- Explicación del fundamento y finalidad del rotavapor: 15 minutos.

- Explicación de la cromatografía en capa fina (ccf) que permitirá el análisis cualitativo de la pureza de los productos aislados: 30 minutos. Se pedirá el día anterior que uno de ellos se prepare esta técnica en casa y la explique en clase. Se valorará positivamente su 
intervención. Se pretende que el alumno participe activamente en las clases.

- Realización de la ccf y visualización mediante lámpara UV: 20 minutos.

- Medida de los puntos de fusión como criterio de pureza de los compuestos aislados de la mezcla: 20 minutos.

- Completar la plantilla ya que ha de ser entregada al finalizar la sesión: 20 minutos.

- Cuestionario final: 15 - 20 minutos

- Desecho de residuos, limpieza del material y del puesto de trabajo: 10 minutos.

- Completar un ejercicio/problema planteado por el profesor mediante un diagrama de separación que servirá de recapitulación de la práctica y que formará parte de la evaluación: 15 minutos. una)

Sintesis de p-nitroanilina (3 sesiones de 3 horas cada

\section{1a Sesión (180 min)}

- Se colocan los alumnos en sus puestos de trabajo: 5 minutos.

- Cuestionario inicial (5 preguntas): 20 minutos.

- Contextualizar la práctica haciendo hincapié en la importancia de las reacciones de nitración en síntesis orgánica ya que el grupo nitro se encuentra presente en estructuras muy interesantes tales como antibióticos: 10 minutos.

- Planteamiento en la pizarra de la secuencia de síntesis completa: 40 minutos.

Esta práctica es muy densa tanto en los aspectos experimentales como en los teóricos. Aparecen conceptos muy interesantes tales como grupo protector, diferentes tipos de reacciones con sus correspondientes mecanismos, diferentes técnicas experimentales, etc...Por lo tanto en esta primera sesión se introduce la práctica de forma general (con los tres pasos de síntesis), pero en ella solamente se 
analizará el mecanismo de formación de acetanilida (primer paso de la secuencia sintética).

- Trabajo experimental: introducción del grupo protector para la obtención de acetanilida: 45 minutos.

- Debate en grupo de la problemática de esta práctica haciendo uso de esquemas de reacción: 20 minutos. El contenido teórico de esta práctica se presta al coloquio-debate, muy enriquecedor en la formación del estudiante (Reflexión-discusión en grupo).

- Completar plantilla: 20 minutos.

- Desecho de residuos, limpieza del material y del puesto de trabajo: 15 minutos.

\section{2a Sesión (180 min)}

- Se colocan los alumnos en sus puestos de trabajo: 5 minutos.

- -Se explicará en la pizarra el mecanismo tanto del segundo como del tercer paso: 30 minutos. (Reflexión-discusión en grupo).

- Trabajo experimental: se llevará a cabo la reacción de nitración de la acetanilida y la reacción de desprotección del grupo acetamido para aislar el producto final: 100 minutos.

- Puesta en común de aspectos prácticos del desarrollo experimental: 20 minutos.

- Completar plantilla: 10 minutos.

- Desecho de residuos, limpieza del material y del puesto de trabajo: 15 minutos.

\section{3a Sesión (180 min)}

- Se colocan los alumnos en sus puestos de trabajo: 5 minutos.

- Se explicará en la pizarra la recristalización como técnica de purificación de compuestos sólidos, se dedica a este apartado un tiempo razonable ya que es la 
primera vez que aparece esta técnica en la asignatura: 20 minutos.

Se pedirá el día anterior que un alumno se la prepare en casa y la explique en clase. Este tipo de participación se valorará positivamente ya que fomenta la formación del estudiante.

- Trabajo experimental: recristalización del producto final en sus puestos de trabajo: 60 minutos.

- Recordatorio de ccf: 10 minutos. Se dedica poco tiempo ya que esta técnica apareció en la práctica anterior.

- Trabajo experimental en sus puestos de trabajo: análisis cualitativo de la pureza del producto sintetizado mediante ccf: 25 minutos.

- Recapitulación por parte del profesor de las tres sesiones haciendo hincapié en los puntos más interesantes: 10 minutos.

- Tiempo para completar las plantillas que han de entregar al finalizar esta tercera sesión: 20 minutos.

- Cuestionario final (5 preguntas): 20 minutos.

- Desecho de residuos, limpieza del material y del puesto de trabajo: 10 minutos.

Sintesis de difenilmetanol (1 sesión de 3 horas)

- Se colocan los alumnos en sus puestos de trabajo: 5 minutos.

- Cuestionario inicial: 15 minutos.

- Contextualizar la práctica, síntesis de un alcohol a partir de un compuesto carbonílico: 10 minutos. Se destacará la importancia de los alcoholes en química orgánica tanto como reactivos, disolventes e intermedios sintéticos versátiles.

- Planteamiento de la síntesis en la pizarra por parte del profesor. Reacción general, reactivos, mecanismo implicado, seguimiento de la reacción mediante ccf y técnicas experimentales que se van a emplear a lo largo del desarrollo de la práctica: 30 minutos. Se 
preguntará continuamente al alumnado y se valorará su participación.

- Trabajo experimental individual en sus puestos de trabajo: 40 minutos.

- Recordatorio en la pizarra de la técnica de la recristalización: 10 minutos. Se empleará menos tiempo ya que esta técnica de purificación ya apareció en la práctica anterior.

- Trabajo experimental: recristalización del producto sintetizado (difenilmetanol): 25 minutos.

- Tiempo para completar la plantilla que se entregará al finalizar esta sesión ya que finaliza la práctica: 15 minutos.

- Resolución de un ejercicio de recapitulación planteado por el profesor que inter-relaciona esta práctica y la anterior: 10 minutos.

- Cuestionario final: 15 minutos.

- Desecho de residuos, limpieza del material y del puesto de trabajo: 5 min.

Se hará hincapié en el tiempo dedicado al final de cada sesión al desecho de residuos generados, a la limpieza del material y del puesto de trabajo como una rutina, ya que así se pretende fomentar la disciplina, la adquisición de hábitos y el orden en el trabajo, aspectos fundamentales a la hora de trabajar en un laboratorio.

A continuación se presenta un esquema resumen del modelo metodológico aplicado de forma general en las 3 prácticas desarrolladas (Figura 2): 


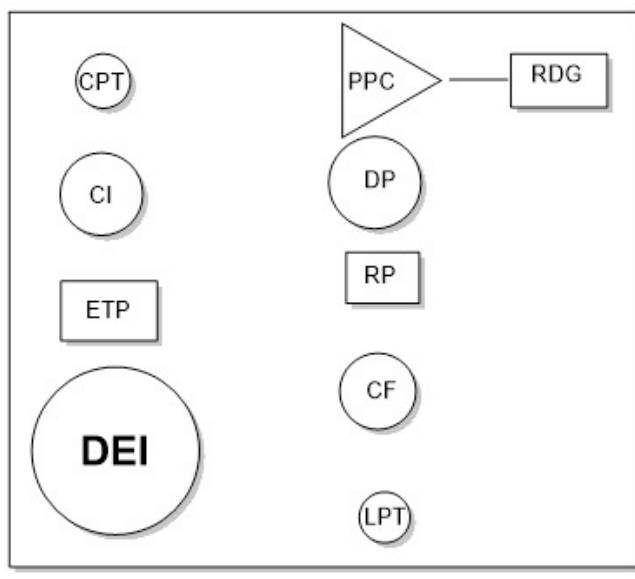

Figura 2. Resumen del modelo metodológico.

En este modelo los círculos representan las actividades realizadas individualmente por el alumno, los rectángulos representan las actividades que se realizan en grupo (discusión, debate, explicación, recapitulación...) y el triángulo representa el trabajo del profesor a través del diseño de preguntas clave.

\section{Aplicación del Ciclo de Mejora Docente}

\section{Relato resumido de las sesiones}

Las sesiones en las que he aplicado los ciclos de mejora han transcurrido sin incidencias. El clima de trabajo ha resultado agradable. Los grupos son reducidos ( 6 alumnos) con lo cual la situación está controlada en todo momento, así como el control de los tiempos que también ha resultado adecuado. Los alumnos parecen estar motivados en el aula, con ganas de abordar la práctica y profundizar en la problemática, sin embargo, el tiempo que le dedican a asimilar los conceptos y a estudiar fuera del aula es muy limitado, cuestiones que parecen haber quedado resueltas

Jornadas de Formación e Innovación Docente del Profesorado I № 1 (2018) Reconocimiento-NoComercial-SinObraDerivada Internacional (CC BY-NC-ND 4.0.) 
en una sesión vuelven a aparecer en sesiones posteriores con muchas lagunas. El docente va guiando al alumno de manera que la práctica pueda ser resuelta satisfactoriamente. El debate/coloquio/conversaciones docente-estudiante y estudiante-estudiante están presentes en todas las sesiones de trabajo, con un constante intercambio entre el profesor y el alumnado.

\section{Evaluación del aprendizaje de los estudiantes}

Para evaluar el aprendizaje de los estudiantes se han determinado 5 niveles de conocimiento para diseñar la escalera:

Nivel A: no sabe absolutamente nada del tema, ideas iniciales nulas.

Nivel B: tiene una idea muy vaga del proceso.

Nivel C: tiene una idea bastante aproximada del proceso pero con lagunas.

Nivel D: conoce bien el tema.

Nivel E: conoce el tema perfectamente.

Para la construcción de la escalera y localizar a los alumnos en el peldaño correspondiente al nivel que presentan tanto en la situación inicial como en la situación final se ha atendido a los resultados obtenidos en las 5 preguntas del cuestionario inicial y final no 2 , correspondiente a una de las prácticas más complejas para el alumnado (Síntesis de p-nitroanilina) (Figura 3).

Jornadas de Formación e Innovación Docente del Profesorado | № 1 (2018) Esta obra se distribuye con la licencia Creative Commons 


\section{$E$}

\section{Situación inicial}

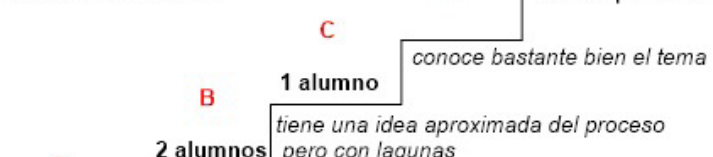

\section{$\mathrm{E}$}

\section{Situación final}

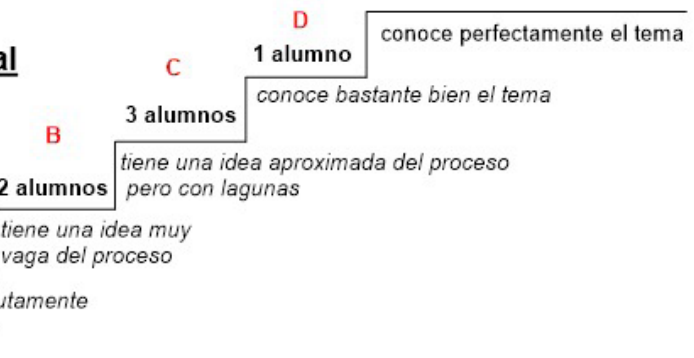

Figura 3. Escalera de evaluación.

Como se observa en las dos escaleras representadas en la Figura 3 hay una clara diferencia entre la situación final y la situación inicial, todos los estudiantes han subido de peldaño. Al principio la mayoría de los alumnos se encontraban en los dos niveles más bajos de la escalera, al finalizar las sesiones ya contamos con 4 alumnos con un cierto nivel, aunque 2 de ellos siguen estando en el nivel $B$ con una idea muy vaga del proceso.

Aunque la situación ideal sería poder colocar a los 6 alumnos en el último peldaño correspondiente al nivel $\mathrm{E}$, pienso que la experiencia ha resultado muy positiva, con una evolución evidente. 


\title{
Evaluación del Ciclo de Mejora Docente
}

\author{
Cuestiones a mantener y cambios a \\ introducir para un futuro Ciclo de Mejora
}

En el desarrollo de este ciclo de mejora docente he tenido la oportunidad de comprobar las diferencias observadas entre los resultados obtenidos en los cuestionarios tipo test y los cuestionarios a desarrollar con respuestas breves realizados el mismo día con el mismo grupo de alumnos. En la tabla 1 se muestra a modo de ejemplo los resultados obtenidos de la práctica Síntesis de $p$-nitroanilina. Con las otras dos prácticas tenemos resultados similares.

Tabla 1

Análisis cuestionarios tipo test/cuestionarios a desarrollar

\begin{tabular}{|c|c|c|}
\hline Alumnos & $\begin{array}{c}\text { Cuestionario inicial } \\
\text { (desarrollo) }\end{array}$ & $\begin{array}{c}\text { Cuestionario inicial } \\
\text { (tipo test) }\end{array}$ \\
\hline Sujeto 1 & 0,5 & 4,0 \\
\hline Sujeto 2 & 0,0 & 4,0 \\
\hline Sujeto 3 & 1,0 & 4,0 \\
\hline Sujeto 4 & 6,0 & 9,0 \\
\hline Sujeto 5 & 3,0 & 4,5 \\
\hline Sujeto 6 & 2,0 & 7,5 \\
\hline
\end{tabular}

Se observa claramente que las notas son más altas cuando el cuestionario es tipo test, pero cuando se evalúa el cuestionario en el que tienen que desarrollar brevemente y poner de manifiesto su conocimiento, los resultados indican claramente que el conocimiento de partida está muy por debajo del conocimiento que indicaba el tipo test. Estos datos sostienen que los cuestionarios tipo test conducen a evaluaciones erróneas, que apoyan resultados engañosos que llevan a confusión. Por tanto, me 
parece muy interesante mantener el cuestionario inicial-final con preguntas clave abiertas incorporándolo en todas las prácticas que comprende la asignatura abandonando definitivamente los cuestionarios tipo test. Mantener el boletín de ejercicios me parece también fundamental para que vayan trabajando casos prácticos al ritmo que crean conveniente que les permita reforzar el contenido teórico de la asignatura.

La incorporación de plantillas (informes dirigidos, propuestos en el proyecto de innovación docente en el que participo) para su resolución en clase al finalizar la práctica que sustituye al informe tradicional me ha parecido muy positiva, con un ambiente de trabajo muy agradable, por tanto, pretendo mantenerlas.

Para un futuro ciclo de mejora me gustaría seleccionar una práctica e intentar dejar al alumno que la resolviera solo, siguiendo su guión, que él la trabajara desde el principio, por supuesto con la supervisión, guía y apoyo del docente. Que la práctica constituyera un problema en sí que ha de ser resuelto en el menor tiempo posible donde el alumno pudiera llevar a cabo el aprendizaje basado en problemas. Si esto funciona, se podría ir aplicando con otras prácticas, de manera que el estudiante se convierta en el responsable de su aprendizaje. Pienso que de esta forma la implicación del alumno sería total, ya que la consecución de la práctica dependería únicamente de él. Esta metodología seguramente requiera más tiempo dedicado a la práctica en cuestión, pero probablemente merezca la pena ya que los conocimientos adquiridos serán más robustos y no se olvidarán tan fácilmente.

\section{Principios didácticos que han guiado la experiencia}

Un aspecto fundamental que ha guiado mi experiencia docente ha sido tratar de establecer interrelación entre todos los contenidos y así intentar transmitírselo a 
los alumnos, que el aprendizaje constituya en sí un conjunto, que el trabajo realizado y los conocimientos adquiridos con trabajo-implicación y esfuerzo no correspondan a experiencias independientes/aisladas. De esta forma se podrá conseguir un conocimiento de la química orgánica duradero y transferible asentado en unos cimientos sólidos y firmes.

\section{Referencias bibliográficas}

Martín Sánchez, M. (2018). Conclusiones de una vida dedicada a la enseñanza. An. Quím., 114, 8-18.

Elguero, J. (2017). Hombres de ciencia y creadores: eso somos los químicos. An. Quím., 113, 218-223.

Bain, K. (2007). Lo que hacen los mejores profesores de Universidad. Valencia: Universitat de Valencia.

Clayden, J., Greeves, N., Warren, S., Wothers, P. (2001). Organic Chemistry. Oxford: University Press.

Palleros, D. R. (2000). Experimental Organic Chemistry. New York: John Wiley \& Sons.

Jornadas de Formación e Innovación Docente del Profesorado | № 1 (2018) Esta obra se distribuye con la licencia Creative Commons 